\title{
Research on Cooperative ADHOC MAC Protocol VANETS Oriented
}

\author{
Sun Yuezhongyi \\ Harbin University of Science and Technology, Harbin, China \\ E-mail:hrb_zl@sina.cn
}

\begin{abstract}
Due to the broad prospect of Vehicular Ad hoc Networks (VANETS) on road safety, traffic management and entertainment applications, data transmission technology of VANETS has been became the focus of the study. However, dynamic topology changes and vehicle move speed of VANETS results in link break of workshop communication, which reduces the reliability of the link. This paper presents the cooperative communication mechanism to improve the reliability of the communication link and ease the wireless road deterioration caused by vehicle movement. Based on the ADHOC MAC scheme, cooperative ADHOC MAC scheme is proposed referred as COOMACP. In COOMACP, once the data packet unsuccessfully transmitted to the destination node, the neighbor nodes will use the unoccupied slot to offer cooperative pattern and retransmit data. Through the theoretical analysis, the probability of cooperative mode is proposed. Theoretical analysis and simulation results show that COOMACP improves the probability of successful transmission of data packets and improves the system throughput.
\end{abstract}

Keywords: Cooperative; Automotive; Wireless Communications; Communication Obstacle; Data Link

\section{Introduction}

Frequent accidents, worsening traffic congestion and strong user demand for Internet access jointly promote the development of Intelligent Transportation Systems (ITS) [1]. ITS can provide a wide range of applications, including improving road safety, traffic efficiency and entertainment. In order to realize these applications, vehicles will be equipped with sensors and communications equipment and form the communications network that is Vehicular ad-hoc network (VANET). In VANET, the vehicle collects information through the advanced sensing and interact information with other vehicles. These vehicles are equipped with an on-board unit (OBU) [2]. Vehicles can realize the function of receive / achieve information through the OBU. Vehicles can also communicate with static equipment side of the road, such as road side facilities (RSU). Therefore, VANETs support the Vehicle-to-Vehicle communication (V2V) and Vehicle-to-Infrastructure communication (V2I) [3-4].

Because of the High-speed mobile nodes and dynamic topology, the traditional wireless network routing protocols are difficult to directly apply to VANETs. For this reason, many scholars pay much attention to the routing scheme applicable to VANETs, such as GSR [4] (Geographic Source Routing) and GPCR [5] (Greedy Perimeter Coordinator Routing). GSR belongs to location-based routing protocol, which utilizes the geographic information as the amount of weight, and calculates the shortest path through the Dijkstra algorithm. Meanwhile, the greedy forward is used to choose the best route. However, GSR does not apply to the node sparse environment. When the node is scarce, there may be not enough nodes to forward packets. STAR also belongs to the location-based routing. Literature [6] proposes the STAR (Spatial and Traffic Aware 
Routing) program. Nodes are required to establish a neighbor table and a traffic table. According to the information in the table, the shortest path is chosen and the packet is greedy forwarded along the shortest path. With performance, STAR is better than GPSR (greedy perimeter stateless routing) [7]; however, STAR consumes more resources than GPSR. GyTAR program [8] (Improved Greedy Traffic Aware Routing) combines the vehicle density information and dynamic obtains the data information of the next anchor. Between the anchors the improved greedy algorithm is used to forward packets. When a packet is forwarded with local optimum, the caching forwarding mechanism is taken. Compared with other wireless networks, in addition to the unreliable and shadow fading outside in wireless transmission network, the implementation of VANETs still faces many challenges. Some strict real-time requirements like fast-moving vehicles, dynamic topology changes and security information add difficulties for the implementation of VANETs. When designing the communication protocol of VANETs these issues must be considered. In order to solve the above problems, the IEEE 802.11p is proposed to be used in the MAC (Medium Access Control) of VANETs. However, the random access channel of IEEE 802.11p causes the without estimated delay and broadcast storms [6-7]. In addition, there are strict real-time constraints of secure message, and when it is necessary there are reliable broadcasting services. Therefore, the TDMA (Time Division Multiple Access) based on MAC protocol is called ADHOC MAC [6], which is used to achieve the reliable broadcast and P2P (Point-to-Point) VANET communication. However, since the dynamic topology of VANET, TDMA MAC protocol may cause the waste of Time slot. When there are not enough neighbor nodes to use intra all time slots, waste of time slots is generated. Moreover, once the data transmission fails, the source node will wait for the next frame to retransmit even if the channel is idle, because there is no available time slot in the frame[12,13]. Accordingly, even if the IEEE 802.11p is used and MAC-based TDMA is under poor channel conditions, it is difficult to overcome the increase of packet loss and the decease of throughput.

Many techniques, such as diversity and channel coding can effectively alleviate the deterioration of the radio channel and improve the network through put [14]. However, these techniques will cause additional overhead or need multiple antennas. The common solution is cooperative communication, which through the nearby neighbor nodes improves the communication performance between the source nodes to the destination nodes. Broadcast nature of wireless transmission makes the neighbor node can receive data packets transmitted from the source nodes to the destination nodes. When the direct transmission between source nodes and the destination nodes is suffered by harsh channel conditions, a neighbor node will forward the packet to destination node with the help of the neighbor nodes. The neighbor nodes help the nodes retransferring data packets to destination nodes are called Helper Node. So for the MAC layer, the cooperative program is proposed, called as CAH-MAC (Cooperative AD HOC MAC). Compared with TDMA and IEEE 802.11p, CAH-MAC uses the distributed TDMA based on MAC protocol. In CAH-MAC, nodes occupy their time slots and neighbor nodes form the clusters and share time frame. Through the collaboration on the link layer, the help node can use the idle time slots to retransmit the packets that failed to reach the destination node. By utilizing the idle slots to forward packets, the proposed CAH-MAC protocol improves the throughput of VANET.

\section{System Model}

In this section, the proposed system model of CAH-MAC protocol is analyzed, including network topology, movement and node distribution. 


\section{A. Network topology and channel model}

It is assumed that in VANET the vehicle moves along the multi-lane; vehicles are randomly distributed; there are $L$ lanes, and the width of each lane is $\omega_{\iota}$; $l \in\{1,2,3, \cdots, L\}$; in the observation period the relative movement of the vehicles is ignored, therefore, it is relative stationary between vehicles. The communication range of vehicle is $r$. Within the communication range of the source nodes, the probability of successful transmission of the received packet is assumed as ${ }^{p}$. Probability ${ }^{p}$ depends on the channel conditions. The smaller the ${ }^{p}$ value is, the worse the channel condition is. Due to the simultaneous transmission of multiple nodes the collision is caused; in this case, $p$ is not reflected.

\section{B. Neighbor Nodes}

Each vehicle maintains a list with one hop or two hops neighbor. One hop or two hops node is the refer node with the transmission distance as one hop or two hops. Accordingly, these neighbor nodes with one hop or two hops are totally called as One-Hop Set, Two-Hop Set. Shown in Figure 1, node A belongs to the two One-hop sets, which respectively are OHS1 and OHS2. In addition, node A also belongs to Two-hop set THS1. A node can directly communicate directly with other nodes within OHS1and OHS2. Similarly, among the nodes within the same THS it is communicated with two-hop.

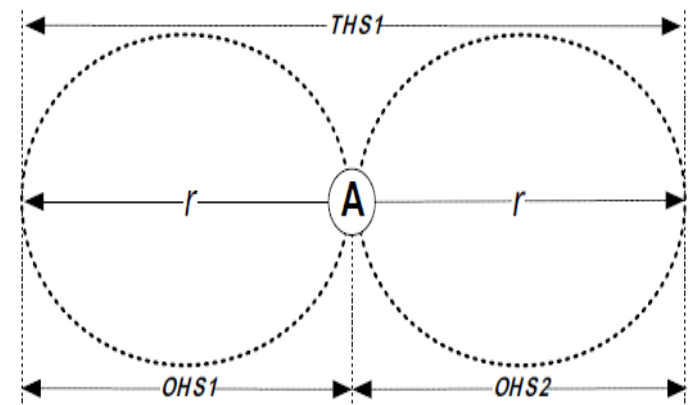

Figure 1. Sample of One-hop and Two-hop

\section{Channel Access}

Based on ADHOC MAC [6] and VeMAC [8], the proposed channel access mechanism is based on a distributed TDMA scheme. By TDMA, channel time is divided into different frames; each frame is further subdivided into time slots. The length of each slot is a fixed period of time; each frame contains a fixed number of time slots denoted as $F$. Each vehicle is able to identify the start of a frame and the start of a time slot. So it is synchronic with the required accurate time between nodes. Global Positioning System (GPS) can be installed in the car to achieve sync. In addition, with the CAH-MAC, the node communication model is consideration as point-to-point. Helper node assists other nodes to retransmit the packet.

Multiple nodes can form two-hop neighbor cluster and the cluster is composed of a group of nodes with the maximum two-hop. There is no cluster head in the cluster, and the node can belong to multiple clusters. The nodes belongs to the same THS will compete for occupying one slot. To get the slot, nodes first spy the channel with $F$ consecutive slots, and then try to occupy a slot. If multiple nodes simultaneously occupy the same slot, the access collision is generated. After it is successful access to the slots, node will transfer packet between the slots of each frame until meet the merging collision 
caused by relative movement [6]. Merging collision is the collision caused by the nodes belong to different clusters while use the time slot [9]. Literature [9] shows that due to the movement of the nodes, throughput is decreased. In order to overcome the decline of throughput, literature [8] proposes the VeMAC program. With the VeMAC, the slots are grouped into three disjoint groups, and three groups are respectively corresponding to the positive and negative direction of the vehicle and the RSU of the vehicle movement. In this way, the problem of decrease of throughput caused by vehicle movement is effectively solved.

The purpose of this study is to improve the transmission reliability, so it is considered that if all the nodes can strictly maintain synchronous and occupy the corresponding time slot, there is no access collisions happened. Also, it ignores the relative movement between the nodes and avoids merging collision.

\section{CAH-MAC Scheme}

This section analyzes the specific programs of CAH-MAC, including the collaborative decision-making choices and assisted selection. Nodes transmit packets in the occupied time slots. This data contains the frame information, cooperation head, header, load data and CRC (Cyclic Redundancy check), as shown in Figure 2,

\begin{tabular}{|c|c|c|c|c|c|c|}
\hline \multicolumn{2}{|c|}{$\begin{array}{c}\text { Frame } \\
\text { Information } \\
\text { (FI) }\end{array}$} & $\begin{array}{c}\text { Cooperation } \\
\text { Header } \\
(\mathrm{COH})\end{array}$ & $\begin{array}{l}\text { Packet } \\
\text { Header }\end{array}$ & \multicolumn{2}{|c|}{ Payload Data } & CRC \\
\hline IDF-1 & IDF-2 & IDF-3 & --- & IDF- $(F-1)$ & DF- $F$ & \\
\hline$i d_{a}$ & $\varphi$ & $i d_{b}$ & --- & $\varphi$ & $i d_{z}$ & \\
\hline
\end{tabular}

Figure 2. Format of Packet

Wherein, CAH-MAC program includes the data header and load data; CRC is the same with ADHOCMAC and VeMAC but different with the frame information. In addition, in the CAH-MAC, the new content is introduced - Cooperation header.

\section{A. Frame Information}

As shown in Figure 2, FI is the set of ID area (IDF). The slot corresponding to each frame IDF, its address is less than 1-2bytes compared with MAC. The short ID makes nodes can be freely selected. Short ID reduces the size of data packet FI and thereby reduces the burden of MAC.

If the destination node $D$ successfully receives the data packet in the time slot $s^{\text {th }}$ from the source node, the time slot $s^{s^{t h}}$ belongs to node ${ }^{S}$. At this time, the node $D$ stores the ID of the node ${ }^{S}$ stored at the $s^{s^{t h}}$ IDF region of FI. Node ${ }^{S}$ successfully receives data packet, and node $D$ can obtain the following information: (a) node $S$ is its one-hop neighbors; (b) node $S$ occupies the time $\operatorname{slot}^{s^{t h}}$; (c) node $S$ belongs to the entire neighbors with one-hop and their corresponding slots. Thus, by successfully received FI information from the one-hop neighbor nodes, the node is to maintain its neighbor node table, including all hop neighbor nodes of (i); (ii) all two-hop neighbor nodes; (iii) time slots occupied by all these nodes. If there is no signal in a slot, there is no occupied slot of this node; in this case, the corresponding area of IDF is empty ${ }^{\phi}$, as shown in IDF-2 2.

\section{B. Cooperation Between Neighboring Nodes}

Cooperation is realized by the one-hop neighbor nodes between source nodes and destination nodes. Since the unoccupied time slots are the same with channel conditions 
of the time slots of the source node, there is no benefit for the source node to retransmit packets in unoccupied time slots, and there is a waste of transmission resources. In other words, in the unoccupied slot, through the independent channels, cooperative forwarding transmission of data packets is achieved to realize the data transmission diversity; thus the source node and the destination node can effectively transmit the data under the harsh channel conditions to improve the reliability of data transmission. Next, nodes' decisions and cooperation are analyzed.

It is supposed that time slot within a frame ${ }^{\Gamma=\{1,2,3, \cdots, F\}}, \mathrm{O}_{x}$ and $\mathrm{T}_{x}$ represent the OHS and THS of node ${ }^{x} \cdot{ }^{\Re} x$ represents all slots of THS belonging to $\mathrm{T}_{x}$. The occupied time slots of the source node $S$ and the destination node $D$ are respectively $s^{s^{t h}}$ and $d^{d^{t h}}$. Node $H$ is the help node. Only when all the following conditions are met, the forward transmission is set up.

(1) Failure of direct transmission

When direct transmission between the source node and the destination node fails, the cooperation is started. Once the transmission fails, the node $D$ is beyond the communication range of the node ${ }^{S}$, i.e. ${ }^{S \notin \mathrm{O}_{D}}$. After receiving FI information from the node $D$, the potential help node can get the failed transmission.

(2) Based on the purpose of retransmission, the help node successfully receives the data packet

Only when node successfully receives the data packets from source node ${ }^{S}$ at time slot $s^{\text {th }}$, the node may make it cooperate and auxiliary transmit data, thereby performing retransmission.

(3)Destination node is within the communication range

When the destination node $D$ can not receive data from the source node $S$, help node $H$ can forward packets to the destination nodes $D$, which indicates that the node $D$ is within the communication range of node ${ }^{H}$. Therefore, node $D$ and ${ }^{S}$ must be the one-hop neighbors of node ${ }^{H}$, namely $S, D \in \mathrm{O}_{H}$.

(4) There is an available time slot

When the condition (1), (2) and (3) are met, if there is at least one available slot $h \in \Gamma$, the help node $H$ can offer cooperation. There is no collision of slot $h$ and transmission $H$ from its neighbor nodes, namely $\forall h \notin \Re_{H}$.

If these conditions are met, the help node ${ }^{H}$ can offer cooperation for source node and destination node, and it can finish the cooperative transmission at $\operatorname{slot}^{h}$. If there are many potential help nodes, the first node answering will forward the packets, and becomes the help node. While there is no need for other potential help nodes dealing with the packet, that is, they will abandon cooperation.

Figure 3 shows that in the process of cooperation with CAH-MAC, there is a exchange of necessary information. When the destination node $D$ can not receive data packets from the source node $S$, as shown in 3 (a), the node $D$ report this message through its neighbor nodes FI shown in Figure 3 (b). After receiving FI information, if cooperation is decided, this node (Assumed as node ${ }^{H}$ ) expresses its willing of cooperation through $\mathrm{COH}$ (Cooperation header), as shown in Figure 3 (c). Within the slot $h^{\text {th }}$, C-ACK (Cooperation acknowledgement) is received from destination node $D$, and the help node $H$ transmits the packet, shown in Figure 3. 


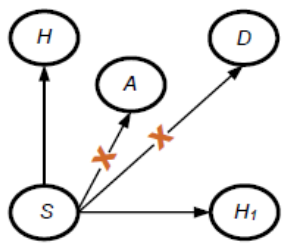

(a)

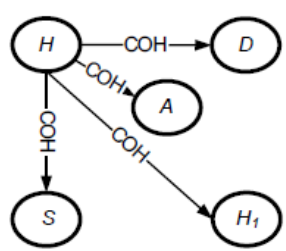

(c)

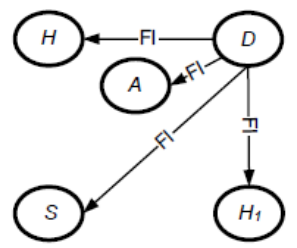

(b)

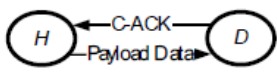

(d)

Figure 3. Information Exchanges Process of CAH-MAC Program on the Cooperation Stage

\section{C. $\mathrm{COH}$ and Cooperation Confirmation}

Once the nodes decide to cooperate, they will pass the cooperation willing through $\mathrm{COH}$ within the packet. $\mathrm{COH}$ contains the following information:

(a) Decisions of cooperation;

(b) Number of slots of the source nodes when the transmission failure occurs;

(c) Number of slots when the help node retransmits data for the destination node

The information above is loaded in $\mathrm{COH}$ and transmitted in the slot of the help nodes. Once the other potential receives cooperation decision from the help node, the cooperation willing is stopped. Thus, the node ${ }^{H}$ becomes the first cooperative node offering service for routing from the source node to the destination node $D$. However, when both the potential help node are beyond the scope of OHS, they may collide at the destination node, so the destination node C-ACK (Cooperation acknowledgement) at the available time slot, as shown in Figure 4. With the C-ACK, the destination node loads the help node ID. Through C-ACK transmission, other potential nodes stop their transmission to avoid collisions.

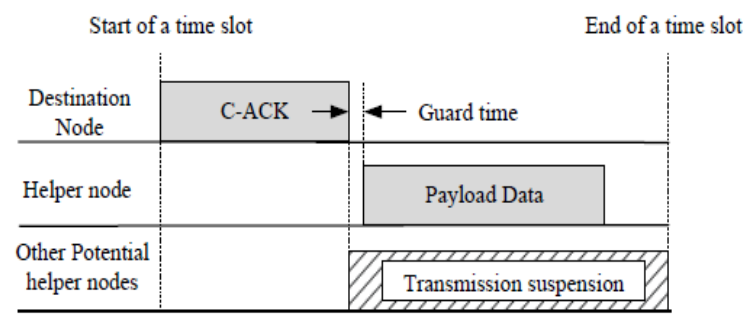

Figure 4. Transmitting Cooperative Acknowledge at Available Time Slot

\section{CAH-MAC Experiments and Analysis}

In order to analyze the performance of CAH-MAC, this section presents the mathematical model to evaluate the performance of CAH-MAC throughput.

\section{A. Node Distribution}

Vehicles are randomly distributed in the driveway. The lane section $\mathrm{o}$ is divided into ${ }^{L}$ segments; for each segment ${ }^{l}$, its vehicle density is expressed as $\rho_{l}$, 
$l \in\{1,2,3, \cdots, L\}$. For a particular road, the number of vehicles is the Poisson process. Therefore, $\mathrm{t}$ at the road with the length of $z$, the probability of finding $m$ vehicles are at following:

$$
p(m, z)=\frac{(p z)^{m} e^{-\rho z}}{m !}, m=0,1,2 \cdots
$$

Wherein $\rho=\sum_{l=1}^{L} \rho_{l}$

\section{B. Distribution of the Number of Neighbor Nodes}

For the given THS, the total number of nodes is $N_{T}$, including the number of nodes of forward, backward and the distance of $r$ shown in Figure 1. As the number of nodes

belongs to the Poisson distribution, PMF (Probability mass function) of $N_{T}$ can be expressed as following:

Next, the number of nodes of the source node to the destination node with the maximum hop is considered and noted as ${ }^{N}$ o. As shown in Figure 5, all the nodes within the OHS of the source node and the destination node are located in the path with the length as $2 r-u . u$ is the distance from the source node to the object program, $u \leq r$. If $u$ is uniformly distributed within ${ }^{[0, r]}$, the average distance from the source node to the object program is as $0.5 r$. Thus, in general the OHS nodes are uniformly distributed in the path as $1.5 r$ lane, i.e. $z=1.5 r$, and the probability mass function of $N_{o}$ is as following:

$$
\operatorname{Pr}\left\{N_{o}=v\right\}=\frac{(1.5 \rho r)^{v} e^{-1.5 \rho r}}{v !}, v=0,1,2 \cdots
$$

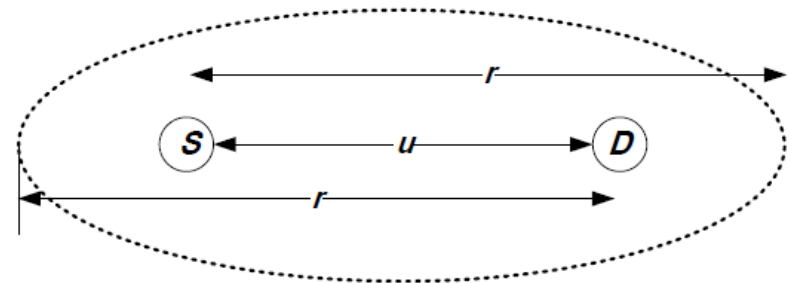

Figure 5. Illustration of the Road Segments from the Source Node to the Destination Node

\section{Slot type and Distribution}

Within the Frame time slot is consisted of unreserved, successful, and failed. Unreserved time slot means that there is no node currently occupied at this slot. Successful time slot refers to the time slot data successfully transmitted from the destination node to the source node. Failed time slot is all timeslots except for UTS and STS.

In the process of cooperative transmission, when the direct transmission is failed, UTS is used to implement the forward transmission. If through the cooperation of the help node, the data has been successfully transferred to the destination node, then UTS became STS; otherwise, the FTS.

The ability dealing with channel errors is used to evaluate the performance of MAC protocol. In the TDMA based on MAC, the number of STS owned by each frame is the parameters to measure their capability. If under the relatively poor channel conditions and MAC protocol data packets can still valid, it indicates that it can get a lot of STS. $p_{s}$ is the probability of successfully transmitting packets in UTS. 


$$
p_{s}=\left(1-p_{c}\right) p
$$

Wherein, ${ }^{p_{c}}$ represents the transmission probability of collision.

According to the literature [10] and literature [11], the desired number of STS can be expressed as $E[X]$ :

$$
\begin{aligned}
E[X]= & p_{s} \sum_{j=1}^{F-1}(F-j) \frac{(2 \rho r)^{F-j} e^{-2 \rho r}}{(F-j) !} \\
& +p_{s} F\left(1-\sum_{j=1}^{F-1} \frac{(2 \rho r)^{j} e^{-2 \rho r}}{j !}\right)
\end{aligned}
$$

\section{Cooperation Start}

If the transfer failed, cooperation will be started. Based on the previous analysis, once the following conditions meet, cooperation will be started.

(1) Event 1 (): There is at least a potential help node.

(2) Event 2 (): There is at least a slot UTS

Event $E_{1}$ depends on the channel conditions of the node from source node to the destination node. The event $E_{2}$ depends on the number of THS of the help node members, and ${ }^{E_{1}}$ and $E_{2}$ are independent. For a route the direct transmission is failed, the cooperation probability $p_{\text {coop }}$ can be expressed as following:

$$
p_{\text {coop }}=\operatorname{Pr}\left\{E_{1}\right\}\left\{E_{2}\right\}
$$

Next, the probability of $E_{1}$ and $E_{2}$ are analyzed.

For two communicating entities of source node and destination node, OHS's number of neighbors obeys the binomial distribution. These nodes are potential help node. It is assume that the random variable $Y$ represents these potential help nodes. The number of nodes within OHS from the source node to the destination node is Set as ${ }^{N_{0}}$, and ${ }^{N_{0}}=k$. If $k \leq 2$, there is no potential secondary node. If $3 \leq k \leq F, k-2$ node is supposed to be the help node. Thus, when ${ }^{N_{0}}=k$, the probability mass function of $Y$ is shown in formula (7) :

$$
\begin{aligned}
& \operatorname{Pr}\left\{Y=a \mid N_{o}=k\right\}= \\
& \left\{\begin{array}{c}
1, \quad \text { for } a=0 \text { if } k \leq 2 \\
\left(\begin{array}{c}
k-2 \\
a
\end{array}\right) p_{s}^{a}\left(1-p_{s}\right)^{k-a-2}, \text { for } 0 \leq a \leq k-2 \text { if } 3 \leq k \leq F \\
\left(\begin{array}{c}
F-2 \\
a
\end{array}\right) p_{s}^{a}\left(1-p_{s}\right)^{F-a-2}, \text { for } 0 \leq a \leq F-2 \text { if } k>F
\end{array}\right.
\end{aligned}
$$

When $Y>0$, event ${ }^{E_{1}}$ occurred. When ${ }^{N_{0}}=k$, the probability of occurrence of an event ${ }^{E_{1}}$ is as following:

$$
\operatorname{Pr}\left\{E_{1} \mid N_{o}=k\right\}=1-\operatorname{Pr}\left\{Y=0 \mid N_{o}=k\right\}
$$

Combined with formula (3) (7) and (8), the probability of occurrence of the event ${ }^{E_{1}}$ is as follows: 


$$
\begin{aligned}
& \operatorname{Pr}\left\{E_{1}\right\}=\sum_{k=3}^{F}\left(1-\left(1-p_{s}\right)^{k-2}\right) \frac{(1.5 \rho r)^{k} e^{-1.5 \rho r}}{k !} \\
& +\left(1-\left(1-p_{s}\right)^{F-2}\right)\left(1-\sum_{k=0}^{F} \frac{(1.5 \rho r)^{k} e^{-1.5 \rho r}}{k !}\right)
\end{aligned}
$$

For event ${ }^{E_{2}}$, if UTS exists within frame, event ${ }^{E_{2}}$ occurs, and the probability of occurrence is as follows:

$$
\operatorname{Pr}\left\{E_{2}\right\}=\sum_{j=1}^{F-1} \frac{(2 \rho r)^{k} e^{-2 \rho r}}{j !}
$$

According to formula (6), (9) and (10), the cooperation probability is calculated as $p_{\text {coop }}$.

It can be found that the start of cooperation does not necessarily guarantee the success retransmissions. Only when the help node forwards the data to the destination node, the cooperation takes effect, and earns benefit. Therefore, cooperative benefit represents the help node successfully forwarded the data, and the probability is $p_{s}^{\text {coop }}$ :

$$
p_{s}^{\text {coop }}=p_{s}+p_{s}\left(1-p_{s}\right) p_{s}^{\text {coop }}
$$

\section{E. Throughput Analysis}

Throughput is defined as the ratio of the number of success slots STS in each frame F and the overall slots. $\sigma$ and ${ }^{\sigma}{ }_{\text {coop }}$ are respectively the throughput of CAH-MAC and ADHOC MAC shown in formula (12).

$$
\left\{\begin{array}{c}
\sigma=\frac{E[X]}{F} \\
\sigma_{\text {coop }}=\frac{E[X]}{F}
\end{array}\right.
$$

Normalized throughput gain is shown in formula (13) as follows:

$$
\sigma_{\text {gain }}=\frac{\sigma_{\text {coop }}-\sigma}{\sigma}
$$

\section{F. Simulation Results}

The simulation is selected following scenarios: a two-way lane ${ }^{L}=2$; lane width is $\omega_{l}=5 \mathrm{~m}$; length is $1000 \mathrm{~m}$. 500 cars are distribution along the driveway with Poisson distribution. The vehicle density $\rho_{l}$ (vehicles / $\mathrm{km}$ ) of two lanes are equal. Next, throughput and throughput gain of ADHOC MAC and CAH-MAC are analyzed.

Figure 6 shows the vehicle density ${ }^{\rho_{l}}$, the number of time slot F, communication radius $\mathrm{R}$ and the impact of different channel $p$ to the throughput. 


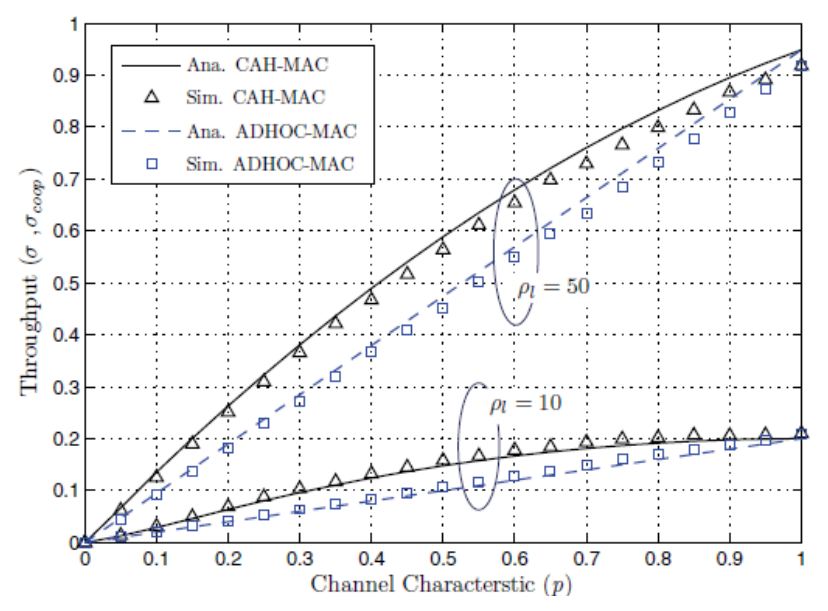

(a) $(r=300 m, \mathbf{F}=60)$

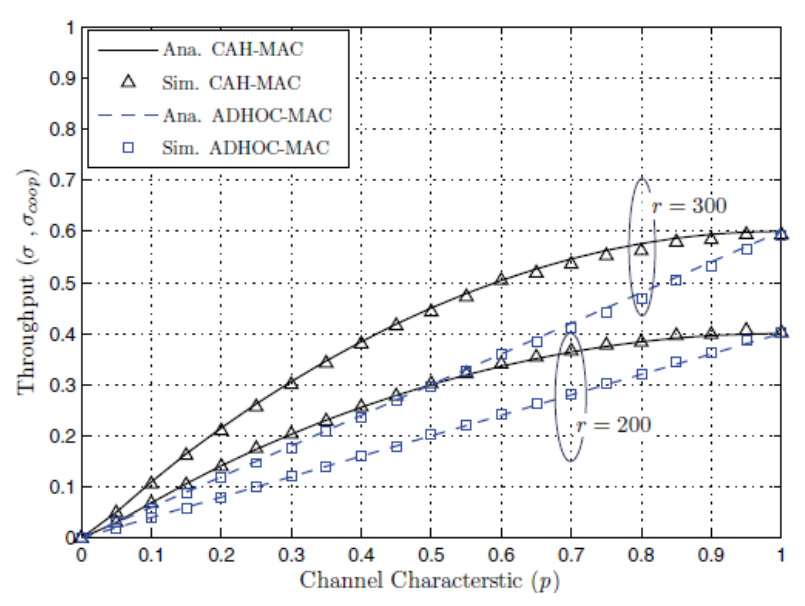

(b) $\quad\left(\rho_{l}=30, \mathbf{F}=60\right)$

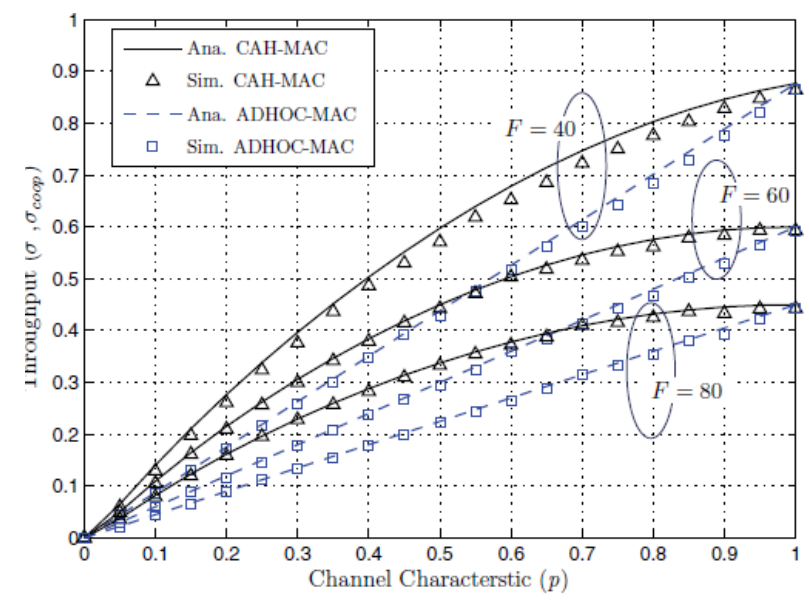

(c) $(r=300, \mathbf{F}=60)$

\section{Figure 6. Changes of Throughput with the Channel characteristic}

Figure 6 (a) shows when $\rho_{l}=10$ and ${ }^{\rho_{l}}=50$, the performance curve of the theoretical analysis and experimental simulation of ADHOC MAC and CAH-MAC with the 
conditions changes, wherein $r=300 \mathrm{~m}$ and the number of slots in each frame is $\mathrm{F}=60$.

Figure 6 shows that the throughput increases with the increase of vehicle density ${ }^{\rho_{l}}$. This is mainly due to the decrease of $\rho_{l}$ means the decrease of the number of members of THS. Compared with ADHOC MAC scheme, CAH-MAC has a higher throughput because CAH-MAC makes full use the UTS time slots and helps nodes are used to cooperatively transfer nodes. In the ADHOC MAC scheme, the number of slots time of UTS is unused to improve the probability of data transmission failure.

As Seen from Figure 6 (b), with the increase of the radius $r$ of the communication the throughput is increased, and the reason is that for the given density of a vehicle, the increase of $r$ add the number of members of the THS, thus each time slot within the frame is better utilized. Figure 6 (c) shows the effect of F on throughput. Under the given circumstances of the vehicle density and communication radius, throughput decreases with the increase of F. For relatively small members THS, the increase of F increases the number of time slots of UTS, thereby the throughput is reduced. The throughput data of ADHOC MAC and CAH MAC with the same channel conditions can be obtained from Figure 6. From the data it can be seen, the throughput of CAH MAC is better than ADHOC MAC, even if the channel conditions are harsh environments. This is mainly because CAH MAC uses the cooperative mechanism of secondary nodes.

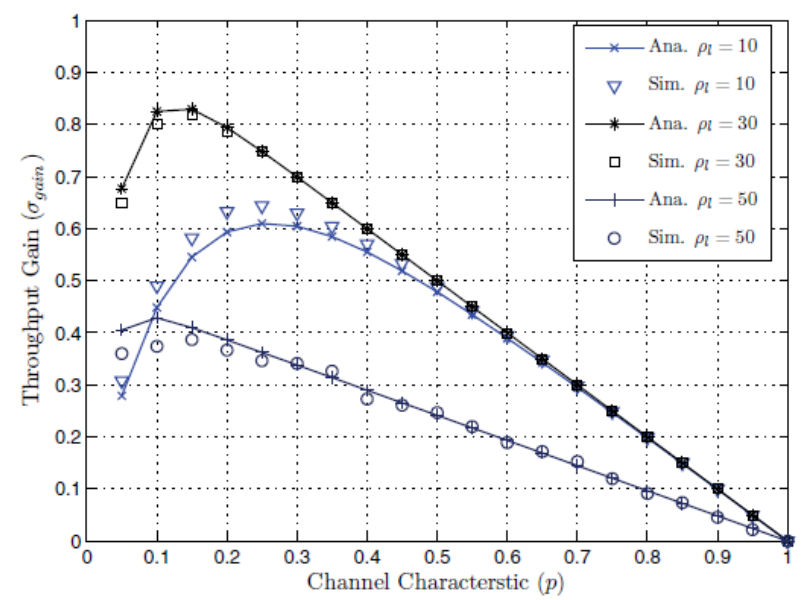

Figure 7. Changes Curve of Throughput Gain with the Channel characteristic

$$
(r=300, \mathbf{F}=60)
$$

Figure 7 shows the performance of throughput gain of CAH-MAC and ADHOC MAC. In the case of vehicle density $\rho_{l}$ changing from 10 to 30 , the throughput gain of CAH-MAC and ADHOC MAC increases with the increase of the channel conditions ${ }^{p}$, because when the vehicle density increases the number of potential help nodes is increased, thereby the probability of occurrence of the event $E_{1}$ is increased. However, since the increase of the number of nodes causes the decrease of the number of slots of UTS, the cooperative benefit is reduced, i.e. the probability of occurrence of the event ${ }^{E_{1}}$ is decreased. Thus, when $\rho_{l}$ is increased from 30 to 50, the throughput gain is decreased.

\section{Conclusion}

Based on the ADHOC MAC scheme, cooperative ADHOC MAC scheme is proposed referred as CAH-MAC. In CAH-MAC, once the data packet unsuccessfully transmitted to the destination node, the neighbor nodes will use the unoccupied slot UTS to offer 
cooperative pattern and retransmit data. UTS is used for retransmission of data to improve the system throughput. In addition, the simple calculation expression of the throughput of CAH-MAC protocol scheme is derived, and then this expression is used for simulation. Simulation data show that the proposed CAH-MAC scheme improves throughput compared with the ADHOC MAC. Meanwhile, CAH-MAC has better throughput gain.

\section{References}

[1] H. Huang, H. Chen, R. Wang, Q. Mao, R. Cheng, "Secret Sharing Scheme Based on Cylinder Model in Wireless Sensor Networks", Journal of Networks, vol. 7, no. 7, (2012), pp. 1009-1016.

[2] United States Department of Transportation. Intelligent transportation systems. [Online]. Available: http://www.its.dot.gov/index.htm

[3] H. Hartenstein, K. P. Laberteaux, "A Tutorial Survey on Vehicular Ad Hoc Networks, IEEE Communications Magazine, vol. 46, no. 6, (2008), pp.164-171.

[4] J. Isaac, S. Zeadally, J. Camara, "Security attacks and solutions for vehicular ad hoc networks", Communications, IET, vol. 4, no. 7, (2010), pp.894-903.

[5] Z. Mengxin, Z. Lv, X. Zhang, G. Chen and K. Zhang, "Research and Application of the 3D Virtual Community Based on WEBVR and RIA," Computer and Information Science 2, no. 1, (2009), p. 84.

[6] S. Tianyun, Z. Lv, S. Gao, X. Li, and H. Lv, "3D seabed: 3D modeling and visualization platform for the seabed." In Multimedia and Expo Workshops (ICMEW), 2014 IEEE International Conference on, (2014), pp. 1-6.

[7] J. Dingde, Z. Xu, P. Zhang, and T. Zhu, "A transform domain-based anomaly detection approach to network-wide traffic." Journal of Network and Computer Applications, vol. 40, (2014), pp. 292-306.

[8] M. Hassan, H. Vu, and T. Sakurai, "Performance analysis of the IEEE 802.11 MAC protocol for DSRC safety applications. IEEE Trans. Veh. Technol., vol. 60, no. 8, (2011) October, pp. 3882-3896.

[9] H. Omar, W. Zhuang, and L. Li, "VeMAC: A TDMA-based MAC protocol for reliable broadcast in VANETs to be published.

[10] Lv. Zhihan, and T. Su, "3D seabed modeling and visualization on ubiquitous context." In SIGGRAPH Asia 2014 Posters, (2014), p. 33. ACM.

[11] L. Zhihan, L. Feng, S. Feng, and H. Li, "Extending Touch-less Interaction on Vision Based Wearable Device." Virtual Reality (VR), (2015), iEEE. IEEE.

[12] H. Zhu and G. Caor, "DCF: A relay-enabled medium access control protocol for wireless ad hoc networks,” IEEE Trans. Mobile Comput.,vol. 5, no. 9, (2000) September, pp. 1201-1214.

[13] Y. Geng, Y. Wan, J. He and K. Pahlavan, "An Empirical Channel Model for the Effect of Human Body on Ray Tracing", 2013 IEEE 24nd International Symposium on Personal Indoor and Mobile Radio Communications (PIMRC), London, Britain (2013) September, pp. 47-52.

[14] Y. Geng, J. He and K. Pahlavan, "Modeling the Effect of Human Body on TOA Based Indoor Human Tracking", International Journal of Wireless Information Networks (IJWIN), vol. 20, no. 4, (2013) December, pp. 306-317.

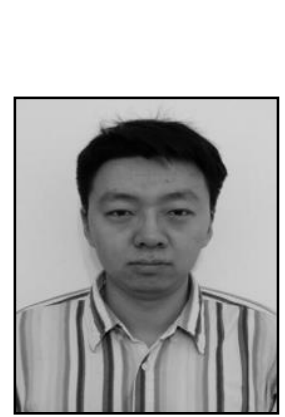

\section{Authors}

Sun Yuezhongyi, male, was born in Harbin. His research Areas: Internet of Vehicles and Mobile Computing. 